\title{
Civilisations
}

Revue internationale d'anthropologie et de sciences

humaines

52-1 | 2004

Transmission des savoirs et interactions culturelles

\section{Transmission des savoirs et interactions culturelles}

Introduction

\section{Paul-Louis van Berg}

\section{(2) OpenEdition}

12 Journals

Édition électronique

URL : http://journals.openedition.org/civilisations/727

DOI : $10.4000 /$ civilisations. 727

ISSN : 2032-0442

Éditeur

Institut de sociologie de l'Université Libre de Bruxelles

Édition imprimée

Date de publication : 1 mai 2004

Pagination : 5-7

ISSN : 0009-8140

\section{Référence électronique}

Paul-Louis van Berg, «Transmission des savoirs et interactions culturelles », Civilisations [En ligne],

52-1 | 2004, mis en ligne le 26 janvier 2009, consulté le 02 juin 2020. URL : http://

journals.openedition.org/civilisations/727 ; DOI : https://doi.org/10.4000/civilisations.727

(c) Tous droits réservés 


\title{
Transmission des savoirs et interactions culturelles
}

\author{
Introduction
}

\section{PAUL-LOUIS VAN BERG}

Ce second volume rassemble les Actes, pour 1998-1999, du Séminaire organisé par le Centre de Recherche interfacultaire « Espaces et Sociétés - approches comparatives » de l'Université Libre de Bruxelles.

Ce Centre est coordonné depuis 1996 par Paul-Louis van Berg (archéologue) et Philippe Jespers (anthropologue), assistés depuis 1998, par Marc Vander Linden (archéologue). Il a pour vocation d'explorer les rapports qui unissent les modalités d'appropriation et d'organisation de l'espace, les formes d'organisation sociale et les idéologies, dans une double perspective, à la fois archéologique et anthropologique. Hors cette orientation générale, les orateurs ne reçurent aucune directive et chacun eut la liberté d'aborder son sujet comme il l'entendait. Nous pensons, en effet, que le contenu factuel d'un exposé ne polarise qu'une petite partie de l'intérêt des auditeurs, tandis que l'apport principal d'un discours tient dans l'approche ou la méthode utilisée, dont chacun peut tirer profit. Il semble que cette manière de concevoir le séminaire ait aussi fait son succès de 1996 à ce jour, puisque, depuis quatre ans, il a réuni chaque mois de vingt à cinquante professeurs, chercheurs, étudiants et personnes intéressées par les problématiques abordées et désireuses d'y réfléchir dans une perspective pluridisciplinaire. On y verra aussi une occasion de pratiquer cette «libre pensée » qui fait la fierté de notre maison.

Comme les années précédentes, le thème de la réflexion et de la recherche a été délibérément choisi suffisamment large pour que chacun y trouve à la fois sa place et son profit, et pour que puisse s'instaurer un dialogue transdisciplinaire. La diversité des spécialités professionnelles des participants et l'assiduité dans la fréquentation du Séminaire ont montré qu'il existait effectivement une demande pour ce genre de débat et la réflexion méthodologique qu'il suppose.

Les travaux de cette année ont porté sur deux thèmes :

1. la transmission des savoirs ;

2. les notions d'espace et d'aire culturelle.

La transmission des savoirs est intimement liée au fonctionnement même des cultures, dans la mesure où elle vise avant tout à en assurer la pérennité. Il s'agit non seulement des contenus, mais des modes de transmission eux-mêmes. Trois enquêtes abordent les problématiques évoquées à partir de sources littéraires ou iconographiques. 
Aller à l'école en Mésopotamie, pour apprendre à lire, écrire et se faire donner les rudiments des sciences d'observation ou recevoir une initiation orale auprès d'un maître indo-européen pour qui le monde visible n'est qu'un trompe-l'œil, sont des démarches différentes nées dans des cultures qui n'ont que peu de choses en commun.

Il y va de l'espace comme de la société et des idéologies. En effet, les modes d'organisation du savoir et ceux des trois classes précédentes sont isomorphes, comme par nécessité. Pour l'écolier mésopotamien, les éléments de base du classement de la réalité tel qu'il apparaît dans une liste lexicographique, sont disposés dans les cases d'une grille virtuelle et ne franchissent pas les limites de celles-ci. Il en va de même des individus. Le tout est verrouillé par des mythes qui justifient l'identité de chaque chose et de chaque être vivant à lui-même, dans l'idée que les dieux ont créé le monde parfait une fois pour toutes.

A l'autre bout de la chaîne, par exemple dans le monde indien ou celtique, les sages affirment que tous les éléments constitutifs de l'univers sont non seulement interconnectés, mais s'interpénètrent, voire se confondent. Il s'ensuit que les catégories de la réalité n'ont pas de limite stricte. L'homme instruit sait ce qui se dissimule derrière des apparences peu fiables, ce qui se cache derrière les paroles prononcées. Qui sait y faire découvre, d'une facette du réel à l'autre, des liens imprévus. La labilité des formes est bien perceptible dans les arts celtiques et le corpus mythologique regorge de récits de métamorphoses. On découvre sans surprise que l'espace social se rapproche d'un espace topologique plus que d'un quadrillage et qu'il n'y a pas de véritable frontière séparant les hommes de dieux pour ainsi dire omniprésents.

En Mésopotamie, les uns et les autres sont traditionnellement séparés par une limite impossible à franchir. Pourtant, appliquée aussi brutalement, une telle démarcation n'est pas viable. Pour que les hommes disposent du savoir nécessaire à leur survie, il faut que les dieux les aient instruits et, en particulier, que le roi ait accès à ce savoir et dispose de spécialistes dans chaque branche. Mythes et institutions illustrent les modalités de cette transmission.

Un peu plus tard, dans le Proche-Orient et l'Egypte hellénisés, plusieurs courants de pensée chercheront à rendre ce contact plus intime et à permettre la remontée des hommes vers les dieux. C'est la solution explorée par les Gnoses de l'Antiquité, comme plus tard par la Kabbale ou par des penseurs mystiques médiévaux tels qu'Avicenne ou Sohravardi. La révélation d'une connaissance initiatique y ouvre des chemins qui permettent à l'âme de remonter vers la divinité. Typologiquement, cette révélation, que nous connaissons par des récits visionnaires, est donnée à des êtres d'exception à l'occasion d'une rencontre avec un personnage supra-humain. Plusieurs problèmes se posent quant à la validation de ce savoir secret. Il s'agit entre autres de le placer dans un monde atemporel et de montrer, en retravaillant les textes sacrés, que cette révélation était donnée depuis toujours mais a été mal comprise, obscurcie et mal transmise. Cette thématique « littéraire » est intimement liée à la cosmologie : manipulations de l'espace et du temps sacrés vont de pair.

Trois enquêtes abordent la question de savoir comment les structures de l'espace s'actualisent sur le terrain. L'une, menée chez les nomades du Turkana montre comment un schéma spatial bipolaire associé à des contraintes écologiques fortes, apparemment, détermine non seulement les déplacements des pasteurs au cours de l'année, mais imprègne aussi le rituel du sacrifice et les comportements individuels. Il fournit une structure permettant de penser en termes polaires de multiples aspects de 
la vie. Il s'agit là d'un de ces facteurs d'homogénéisation de la culture, processus sur lequel nous insistions l'année dernière. Les isomorphismes qu'il engendre donnent à la culture sa cohérence et son style à la pensée qu'ils aident à organiser. Le schéma trifonctionnel mis en lumière par Georges Dumézil joue, mutatis mutandis, un rôle analogue dans les cultures qui s'expriment en langues indo-européennes.

La possibilité d'aborder le savoir et les idéologies dépend aussi de l'état de la recherche et de la nature de la documentation. Dans les situations préhistoriques, comme c'est le cas dans les Andes centrales du $2^{\mathrm{e}}$ millénaire avant notre ère au $1^{\mathrm{er}}$ millénaire de notre ère, lorsque font défaut les textes et les données linguistiques, c'est la définition même des entités en jeu qui pose problème aux archéologues, pour ce qui est de leur nature, de leur extension territoriale et de leurs interactions. Regardée de près, la documentation impose de rejeter les explications trop générales par le déterminisme écologique ou la reconnaissance trop prompte de cultures homogènes. Elle nous rappelle aussi que les frontières peuvent être tantôt d'infrangibles limites, tantôt l'espace où se déroulent de puissantes interactions et que les communautés, selon les circonstances politiques, peuvent se fondre dans une culture globale ou, au contraire, se replier sur elles-mêmes et faire surgir des identités diversifiées. Les enseignements de l'organisation de l'espace (décor céramique et architecture) quant aux sociétés et à leurs idéologies seraient peut-être une aide précieuse en ce domaine.

Les phénomènes de succession temporelle peuvent être aussi difficiles à conceptualiser que les transitions spatiales. Les attestations archéologiques du passage des cultures mésolithiques de chasseurs aux cultures des premiers paysans néolithiques dans les Balkans ne le cèdent guère en complexité à la problématique précédente. Apport de nouvelles populations, vagues d'arrivées successives, présence/absence de contacts avec les populations locales, rôle de ces dernières dans la diffusion du Néolithique, effets d'interactions diversifiées, adaptations des nouvelles populations aux matières premières trouvées sur place, nous emmènent loin des modèles généralisants. En changeant de niveau d'échelle, c'est-à-dire en allant voir sur le terrain comment les choses se passent, on découvre, comme dans les Andes, une variabilité insoupçonnée. C'est peut-être à cette variabilité qu'il faut s'attendre dès qu'on observe de près un système à mélange. La direction globale du changement est nettement perceptible, mais le mouvement de chaque molécule est localement imprévisible.

Paul-Louis van Berg, Professeur à l'Université Libre de Bruxelles. 